\title{
Introduction: Spinal Vascular Malformations
}

\author{
Edward H. Oldfield, M.D. \\ University of Virginia Health Systems, Charlottesville, Virginia
}

\begin{abstract}
$\mathrm{F}$ ROM the earliest observations of spinal vascular malformations, successful management has been challenging. Initially the challenges were diagnosing, understanding, and treating these lesions. They were originally considered all to be the same, or at least to be a single general type, of disease. With the introduction of selective spinal arteriography in the 1960s and more recently with the introduction and widespread use of MR imaging, the initial challenge of diagnosing spinal vascular malformations was overcome, and significant progress has been made in understanding their anatomy as well as the pathophysiology that underlies the myelopathy commonly associated with them. The anatomical features defined by selective arteriography and the observations permitted with the operating microscope ultimately led to distinctions between the major categories of the vascular lesions affecting the spinal cord; these distinctions were based on the lesions' anatomy, epidemiology, and the mechanism of spinal cord injury.
\end{abstract}

Today the most common type of spinal vascular abnormality, the dural arteriovenous fistula (DAVF), can always be eliminated with successful treatment, although whether that should best be done initially with surgery or with embolic occlusion is still controversial, as is evident by the differing views in several of the papers in this issue of Neurosurgical Focus. Despite the fact that DAVFs can be successfully obliterated, many patients are left with neurological deficits or chronic pain, both of which can be avoided in many cases if the diagnosis is established earlier and therapy delivered promptly. Thus, the remaining important challenge is simply to make the diagnosis earlier.

This issue of Neurosurgical Focus begins with a paper in which the authors review the life and contributions of Albert Adamkiewicz, about whom relatively little has been published; this is followed by a review of the history of the path to recognizing and understanding spinal DAVFs. These articles are followed by a review of the unusual features associated with DAVFs in the cervical region, including emphasis on the importance of including such lesions in the differential diagnosis of subarachnoid hemorrhage and an emphasis on pitfalls that should be avoided to ensure their diagnosis. Further details of the clinical features associated with cervical DAVFs and pearls for successful diagnosis and management are included in an accompanying podcast with Dr. William Couldwell.

The literature regarding spinal extradural AVFs with intradural medullary venous drainage (lesions that share a similar clinical presentation with spinal DAVFs) has been limited to case reports. Thus, our understanding of the diverse arterial and venous anatomy associated with extradural AVFs has been as limited as the evidence of how best to approach them for treatment. For the first time, Clarke and colleagues describe a series of patients with extradural AVFs that caused progressive myelopa- thy, and the authors discuss the clinical findings, diagnostic evaluation, treatment, and outcome of this rare entity. Additional material related to this report is provided by Dr. David Piepgras in an accompanying podcast .

As experience has grown in the surgical and embolic treatment of the various types of spinal arteriovenous malformations (AVMs), new approaches have been introduced. This has been particularly true for embolic occlusion, either used alone or followed by surgery. Advances in the embolic materials-initially limited to inert particles of metal or plastic but then followed by the development of glues with various features - provide cause for enthusiasm for the belief that certain challenging AVMs, for which we have no completely effective therapies today, may be successfully treated in the future. One of the byproducts of these advances, however, is that it has been difficult to fully assess the potential of any of them because, before any specific agent can be submitted to a serious study with long-term clinical and arteriographic follow-up, we have already moved on to the next generation, or even the generation after that, of embolic material. Nonetheless, progress has been made, progress that is summarized by the 3 reports examining the use of embolic treatment for the various types of spinal AVMs.

In the penultimate article we look toward the future in a report on the development of noninvasive imaging to diagnose spinal AVM and to evaluate the success of treatments, and in the final article we examine change in national trends in caring for patients with spinal AVMs during the past decade.

There is a wealth of information in these reports, some of it critically reviewing existing knowledge, some of it presenting new material. I hope it is useful to you and that you enjoy spending some time with this issue of Neurosurgical Focus. 